\title{
Ordered extreme ranked set sampling and its application in parametric estimation
}

\author{
Manoj Chacko \\ Department of Statistics, University of Kerala, Kariavattom Campus \\ Trivandrum, Kerala -695581, India \\ manojchacko02@gmail.com
}

Received 8 April 2015

Accepted 1 March 2016

\begin{abstract}
Ranked set sampling (RSS) is applicable whenever ranking of a set of sampling units can be done easily by a judgment method or based on the measurement of an auxiliary variable which can be measured easily. In this work, a modification of RSS called ordered extreme ranked set sampling is considered for Morgenstern family of distributions, in which an auxiliary variable $\mathrm{X}$ correlated with the study variate $\mathrm{Y}$ is used to rank the sample units. This modification of RSS is applied to obtain an estimator of the parameter associated with the study variate $\mathrm{Y}$, when $(\mathrm{X}, \mathrm{Y})$ follows a Morgenstern type bivariate exponential distribution.
\end{abstract}

Keywords: Ranked set sampling, Concomitants of order statistics, Morgenstern family of distributions, Best linear unbiased estimator.

\section{Introduction}

The concept of ranked set sampling (RSS) was first introduced by [11] as a process of improving the precision of the sample mean as an estimator of the population mean. The procedure involves randomly choose $n$ sets of size $n$ each from a population, then the units in each set are ranked visually or using some methods that has negligible cost. From the first set of $n$ units the unit ranked lowest is chosen for actual quantification. From the second set of $n$ units the unit ranked second lowest is chosen for actual quantification. The process is continued until the units ranked highest is chosen from the $n$th set for actual quantification. Then make measurements on the variable of interest of the selected units.

Ranked set sampling as described in [11] is applicable whenever ranking of a set of sampling units can be done easily by a judgment method. Suppose the variable of interest say $Y$, is difficult or much expensive to measure, but an auxiliary variable $X$ correlated with $Y$ is readily measurable and can be ordered exactly. In this case as an alternative to [11] method of ranked set sampling, [13] used an auxiliary variable for the ranking of the sampling units. The procedure of ranked set sampling described by [13] using auxiliary variate is as follows. Choose $n^{2}$ independent units, arrange them randomly into $n$ sets each with $n$ units and observe the value of the auxiliary variable $X$ on each of these units. In the first set, that unit for which the measurement on the auxiliary variable is the smallest is chosen. In the second set, that unit for which the measurement on the auxiliary variable 
is the second smallest is chosen. The procedure is repeated until in the last set, that unit for which the measurement on the auxiliary variable is the largest is chosen.

[14] introduced a modified ranked set sampling procedure called extreme ranked set sampling in which only the largest or the smallest judgment ranked unit is chosen for quantification. The procedure of extreme ranked set sampling using auxiliary variable is as follows. Choose $n^{2}$ independent units, arrange them randomly into $n$ sets each with $n$ units and observe the value of the auxiliary variable $X$ on each of these units. Then from each set choose that unit for with the measurement on the auxiliary variable is smallest or largest, as the unit of the ranked set sample. Then we make measurement on the study variate $Y$ of the selected units, which constitute the extreme ranked set sample (ERSS). If we choose that unit for which the measurement on the auxiliary variable is smallest as the unit of the ERSS then the sample is called Lower Extreme Ranked Set Sample (LERSS). If we choose that unit for which the measurement on the auxiliary variable is largest as the unit of the ERSS then the sample is Upper Extreme Ranked Set Sample (UERSS). Since the units are chosen from independent sets and from each set we choose that unit for with the measurement on the auxiliary variable is either smallest or largest, the observations of ERSS are independently and identically distributed.

[15] considered the estimation of parameters of location-scale family of distributions using RSS. [9], [10] obtained the BLUEs of location and scale parameters of exponential distribution and logistic distribution. [3] obtained an estimator of the mean of the study variate $Y$, when an auxiliary variable $X$ is used for ranking the sample units, under the assumption that $(X, Y)$ follows a bivariate normal distribution. [4], [6] obtained the estimators for the parameters associated with the study variate $Y$, when an auxiliary variable $X$ is used for ranking the sample units, under the assumption that $(X, Y)$ follows a bivariate Pareto distribution and Morgenstern type bivariate logistic distribution. [5] used ERSS to estimate a parameter involved in Morgenstern Type Bivariate Exponential Distribution (MTBED).

In modeling bivariate data, when the prior information is in the form of marginal distributions, it is of advantage to consider families of bivariate distributions with specified marginals. The Morgenstern family of distributions (MFD) is characterized by specified marginals and an association parameter. A bivariate random variable $(X, Y)$ is said to follow a MFD if its pdf is of the form

$$
f(x, y)=f_{X}(x) f_{Y}(y)\left\{1+\alpha\left(1-2 F_{X}(x)\right)\left(1-2 F_{Y}(y)\right)\right\},-1 \leq \alpha \leq 1 .
$$

where $F(x)$ and $F(y)$ are marginal cdfs of $X$ and $Y$ with pdfs $f(x)$ and $f(y)$ respectively. [12] obtained the density function of concomitant of $r$ th order statistic arising from MFD with density function is defined in (1.1) and is given by

$$
f_{[r]}(y)=f_{Y}(y)\left\{1+\alpha \frac{n-2 r+1}{n+1}\left(1-2 F_{Y}(y)\right)\right\},-1 \leq \alpha \leq 1 .
$$

In this work, we introduce a new concept called ordered extreme ranked set sampling in which we arrange the units of the ERSS in ascending order of magnitude. In section 2, we derive the distribution theory of ordered extreme ranked set sample (OERSS) arising from Morgenstren Family of Distributions. In section 3, we apply the distribution theory developed in section 2 to estimate a parameter involved in Morgenstern type bivariate exponential distribution, which is a member of MFD, based on OERSS. Section 4 is devoted to some concluding remarks. 


\section{OERSS from MFD}

Let $X_{(n) i}$ be the observation measured on the auxiliary variate $X$ in the $i$ th unit of the UERSS and let $Y_{[n] i}$ be the measurement made on the $Y$ variate of the same unit, $i=1,2, \cdots, n$. Then it is easy to see that each $Y_{[n] i}$ is the concomitant of the largest order statistic of $n$ independently and identically distributed bivariate random variables with MFD. Moreover $Y_{[n] i}, i=1,2, \ldots, n$ are also independently distributed. The pdf of $Y_{[n] i}$ is obtained by putting $r=n$ in (1.2) and is by

$$
f_{[n]}(y)=f_{Y}(y)\left\{1-\alpha\left(\frac{n-1}{n+1}\right)\left(1-2 F_{Y}(y)\right)\right\} .
$$

The above pdf can be rewritten as

$$
f_{[n]}(y)=f_{Y}(y)\left\{1+\alpha_{n}\left(1-2 F_{Y}(y)\right)\right\}
$$

where $\alpha_{n}=-\alpha\left(\frac{n-1}{n+1}\right)$. Then we order the observations $Y_{[n] i}, i=1,2, \ldots, n$ in ascending order of magnitude to get the ordered upper extreme ranked set sample (OUERSS). Let $Y_{r:[n]}, r=1,2, \ldots, n$ be the observations of OUERSS, then $Y_{r:[n]}$ is the $r t h$ order statistic of a random sample of size $n$ arising from a population with pdf (2.1). In the following theorem we express the pdf of the $r t h$ observation of the OUERSS and joint pdf of $r t h$ and sth observations of the OUERSS arising from MFD in terms of the pdf for the order statistics from the marginal distribution of Y.

Theorem 2.1. Let $Y_{r:[n]}, r=1,2, \ldots, n$ be the OUERSS observations arising from MFD with $p d f$ defined in (1.1). Then the pdf of $Y_{r:[n]}, 1 \leq r \leq n$ is given by,

$$
\begin{aligned}
f_{r: n}(y)= & \frac{n !}{(r-1) !(n-r) !} \sum_{k=0}^{n-r} \sum_{l=0}^{r+k-1}(-1)^{k+l}\left(\begin{array}{c}
n-r \\
k
\end{array}\right)\left(\begin{array}{c}
r+k-1 \\
l
\end{array}\right) \\
& {\left[\alpha_{n}^{l} \beta(r+k, l+1) f_{r+k: r+k+l}(y)+\alpha_{n}^{l+1} \beta(r+k+1, l+1) f_{r+k+1: r+k+l+1}(y)\right.} \\
& \left.+\alpha_{n}^{l+1} \beta(r+k, l+1) f_{r+k: r+k+l+1}(y)\right]
\end{aligned}
$$

and the joint pdf of $Y_{r:[n]}$ and $Y_{s:[n]}$ is given by, for $1 \leq r<s \leq n$ 


$$
\begin{aligned}
& f_{r, s: n}(x, y)=\frac{n !}{(r-1) !(s-r-1) !(n-r) !} \sum_{k=0}^{s-r-1} \sum_{m=0}^{k} \sum_{l=0}^{r-1} \sum_{t=0}^{n-s}(-1)^{l+k+m} \\
& \left(\begin{array}{c}
s-r-1 \\
k
\end{array}\right)\left(\begin{array}{c}
k \\
m
\end{array}\right)\left(\begin{array}{c}
r-1 \\
l
\end{array}\right)\left(\begin{array}{c}
n-s \\
t
\end{array}\right) \\
& {\left[\sum_{p=0}^{l+m} \sum_{q=0}^{k-m+t}(-1)^{p+q}\left(\begin{array}{c}
l+m \\
p
\end{array}\right)\left(\begin{array}{c}
k-m+t \\
q
\end{array}\right) \alpha_{n}^{l+k+t}\left(1-\alpha_{n}\right)\right.} \\
& \frac{(r+m+p-1) !(s-r-k-1) !(n-s-m+q+k) !}{(n+p+q) !} \\
& \times\left(\left(1-\alpha_{n}\right) f_{r+m+p, s+m+p-k: n+p+q}(x, y)\right. \\
& \left.+2 \alpha_{n} \frac{r+m+p}{n+p+q+1} f_{r+m+p+1, s+m+p-k: n+p+q+1}(x, y)\right) \\
& +\sum_{p=0}^{l+m} \sum_{q=0}^{k-m+t+1}(-1)^{p+q}\left(\begin{array}{c}
l+m \\
p
\end{array}\right)\left(\begin{array}{c}
k-m+t+1 \\
q
\end{array}\right) 2 \alpha_{n} \\
& \frac{(r+m+p-1) !(s-r-k-1) !(n-s-m+q+k) !}{(n+p+q) !} \\
& \times\left(\left(1-\alpha_{n}\right) f_{r+m+p, s+m+p-k: n+p+q}(x, y)\right. \\
& \left.\left.+2 \alpha_{n} \frac{r+m+p}{n+p+q+1} f_{r+m+p+1, s+m+p-k: n+p+q+1}(x, y)\right)\right],
\end{aligned}
$$

where $f_{r: n}(y)$ is the pdf of rth order statistic and $f_{r, s: n}(x, y)$ is the joint pdf of rth and sth order statistics of a random sample of size $n$ arising from a population with $p d f f_{Y}(y), \beta(.,$.$) is the usual$ beta function.

Proof. The distribution function corresponding to the pdf $f_{[n]}(y)$ given in (2.1) can be obtained as

$$
F_{[n]}(y)=F_{Y}(y)\left\{1+\alpha_{n}\left(1-F_{Y}(y)\right)\right\} .
$$

Since $Y_{r:[n]}$ is the $r$ th order statistic of a random sample of size $n$ from the distribution with pdf $f_{[n]}$ given in (2.1) and cdf $F_{[n]}$ given in (2.4), the density function of $Y_{r:[n]}$ is given by

$$
\begin{aligned}
f_{r: n}(y) & =\frac{n !}{(r-1) !(n-r) !}\left[F_{[n]}(y)\right]^{r-1}\left[1-F_{[n]}(y)\right]^{r-1} f_{[n]}(y) \\
& =\frac{n !}{(r-1) !(n-r) !} \sum_{k=0}^{n-r}(-1)^{k}\left(\begin{array}{c}
n-r \\
k
\end{array}\right)\left[F_{[n]}(y)\right]^{r+k-1} f_{[n]}(y)
\end{aligned}
$$

On substituting the value of $F_{[n]}(y)$ and $f_{[n]}(y)$ and using binomial expansion we get the pdf (2.2). The joint pdf of $Y_{r:[n]}$ and $Y_{s:[n]}$ is given by

$$
\begin{aligned}
f_{r, s: n}(x, y)= & \frac{n !}{(r-1) !(s-r-1) !(n-r) !}\left[F_{[n]}(x)\right]^{r-1}\left[F_{[n]}(y)-F_{[n]}(x)\right]^{s-r-1} \\
& {\left[1-F_{[n]}(y)\right]^{r-1} f_{[n]}(x) f_{[n]}(y) . }
\end{aligned}
$$


Again by substituting the values of $F_{[n]}(x), F_{[n]}(y), f_{[n]}(x)$ and $f_{[n]}(y)$ and using binomial expansions we get the pdf (2.3)

Next we obtain the moments and product moments of $Y_{r:[n]}, r=1,2, \ldots, n$ as given in the following theorem.

Theorem 2.2. Let $Y_{r:[n]}, r=1,2, \ldots, n$ be the OUERSS observations arising from MFD with $p d f$ defined in (1.1). If we denote $\mu_{r:[n]}^{(q)}=E\left[Y_{r:[n]}^{q}\right]$ and $\mu_{r, s:[n]}=E\left[Y_{r:[n]} Y_{s:[n]}\right]$ then

$$
\begin{aligned}
\mu_{r:[n]}^{(q)}= & \frac{n !}{(r-1) !(n-r) !} \sum_{k=0}^{n-r} \sum_{l=0}^{r+k+1}(-1)^{k+l}\left(\begin{array}{c}
n-r \\
k
\end{array}\right)\left(\begin{array}{c}
r+k-1 \\
l
\end{array}\right) \\
& {\left[\alpha_{n}^{l} \beta(r+k, l+1) \mu_{r+k: r+k+l}^{(q)}+\alpha_{n}^{l+1} \beta(r+k+1, l+1) \mu_{r+k+1: r+k+l+1}^{(q)}\right.} \\
& \left.+\alpha_{n}^{l+1} \beta(r+k, l+1) \mu_{r+k: r+k+l+1}^{(q)}\right]
\end{aligned}
$$

and

$$
\begin{aligned}
& \mu_{r, s:[n]}=\frac{n !}{(r-1) !(s-r-1) !(n-r) !} \sum_{k=0}^{s-r-1} \sum_{m=0}^{k} \sum_{l=0}^{r-1} \sum_{t=0}^{n-s}(-1)^{l+k+m} \\
& \left(\begin{array}{c}
s-r-1 \\
k
\end{array}\right)\left(\begin{array}{c}
k \\
m
\end{array}\right)\left(\begin{array}{c}
r-1 \\
l
\end{array}\right)\left(\begin{array}{c}
n-s \\
t
\end{array}\right) \\
& {\left[\sum_{p=0}^{l+m} \sum_{q=0}^{k-m+t}(-1)^{p+q}\left(\begin{array}{c}
l+m \\
p
\end{array}\right)\left(\begin{array}{c}
k-m+t \\
q
\end{array}\right) \alpha_{n}^{l+k+t}\left(1-\alpha_{n}\right)\right.} \\
& \frac{(r+m+p-1) !(s-r-k-1) !(n-s-m+q+k) !}{(n+p+q) !} \\
& \times\left(\left(1-\alpha_{n}\right) \mu_{r+m+p, s+m+p-k: n+p+q}\right. \\
& \left.+2 \alpha_{n} \frac{r+m+p}{n+p+q+1} \mu_{r+m+p+1, s+m+p-k: n+p+q+1}\right)
\end{aligned}
$$

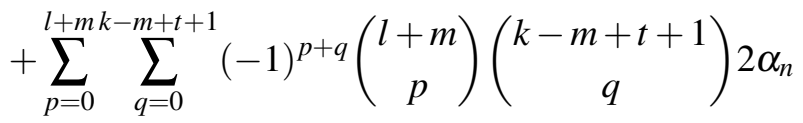

$$
\begin{aligned}
& \frac{(r+m+p-1) !(s-r-k-1) !(n-s-m+q+k) !}{(n+p+q) !} \\
& \times\left(\left(1-\alpha_{n}\right) \mu_{r+m+p, s+m+p-k: n+p+q}\right. \\
& \left.\left.+2 \alpha_{n} \frac{r+m+p}{n+p+q+1} \mu_{r+m+p+1, s+m+p-k: n+p+q+1}\right)\right],
\end{aligned}
$$

where $\mu_{r: n}^{(q)}$ is the qth moment of the rth order statistic and $\mu_{r, s: n}$ is the product moment of $r$ th and sth order statistics of a random sample of size $n$ arising from a population with $p d f f(x)$.

Next we consider the lower extreme ranked set sampling in which the measurement on the auxiliary variable is smallest as the unit of the ERSS. Let $X_{(1) i}$ be the observation measured on the auxiliary variate $X$ in the $i$ th unit of the LERSS and $Y_{[1] i}$ be the measurement made on the $Y$ variate of the same unit, $i=1,2, \cdots, n$. Then each $Y_{[1] i}$ is the concomitant of the smallest order statistic of $n$ 
independently and identically distributed bivariate random variables with MFD and the pdf of $Y_{[1] i}$ is obtained by put $r=1$ in (1.2). Also $Y_{[1] i}, i=1,2, \ldots, n$ are independently distributed with pdf given by

$$
f_{[1]}(y)=f_{Y}(y)\left\{1+\alpha\left(\frac{n-1}{n+1}\right)\left(1-2 F_{Y}(y)\right)\right\} .
$$

The above pdf can be rewrite as

$$
f_{[1]}(y)=f_{Y}(y)\left\{1+\alpha_{n}^{\prime}\left(1-2 F_{Y}(y)\right)\right\},
$$

where $\alpha_{n}^{\prime}=-\alpha_{n}$.

Remark 2.1. The pdf of $Y_{[n] i}$ defined in (2.1) and pdf of $Y_{[1] i}$ defined in (2.9) are similar except for $\alpha_{n}$ changed to $\alpha_{n}^{\prime}$ in (2.9). Hence the pdfs and moments of OLERSS can be easily obtained from the pdfs and moments of OUERSS by replacing $\alpha_{n}$ with $\alpha_{n}^{\prime}$.

\section{Estimation of a parameter of MTBED}

In this section we are trying to estimate the mean of the population, under a situation where in measurement of observations are strenuous and expensive, using OERSS. [2] has proposed an exponential distribution for the study variate $Y$, the oil pollution of the sea samples. Let the auxiliary variable $X$ represents the tar deposit in the nearby sea shore. Clearly collecting sea water sample and measuring the oil pollution in it is strenuous and expensive. However the prevalence of pollution in the sea water is much reflected by the tar deposit in the surrounding terminal sea shore. Thus ranking the pollution level of sea water based on the tar deposit in the sea shore is more natural and scientific than ranking it visually or by judgment method. Hence in this section we assume a Morgenstern type bivariate exponential distribution (MTBED), which is a member of MFD, corresponding to a bivariate random variable $(X, Y)$, where $X$ denote the auxiliary variable (such as tar deposit in the sea shore) and $Y$ denote the study variable (such as the oil pollution in the sea water) with given by (see, [8])

$$
f(x, y)= \begin{cases}\frac{1}{\theta_{1} \theta_{2}} \exp \left\{\frac{-x}{\theta_{1}}+\frac{-y}{\theta_{2}}\right\} & {\left[1+\alpha\left(1-2 \exp \left\{\frac{-x}{\theta_{1}}\right\}\right)\left(1-2 \exp \left\{\frac{-y}{\theta_{2}}\right\}\right)\right],} \\ 0, \text { otherwise. } & x>0, y>0 ;-1 \leq \alpha \leq 1 ; \theta_{1}>0, \theta_{2}>0\end{cases}
$$

Clearly the marginal distributions of $X$ and $Y$ are exponential distributions with pdfs

$$
\begin{aligned}
& f(x)=\frac{1}{\theta_{1}} \exp \left\{\frac{-x}{\theta_{1}}\right\} \\
& f(y)=\frac{1}{\theta_{2}} \exp \left\{\frac{-y}{\theta_{2}}\right\} .
\end{aligned}
$$

[1] have shown that in a bivariate sample of size $n$ arising from MTBED the concomitant of largest order statistic possess the maximum Fisher information on $\theta_{2}$ whenever $\alpha>0$ and the concomitant 
of smallest order statistic possess the maximum Fisher information on $\theta_{2}$ whenever $\alpha<0$. Hence in this section, first we consider $\alpha>0$ and carry out an OUERSS such that from each set we choose an unit of a sample with the largest value on the auxiliary variable as the units of ranked sets with an objective of exploiting the maximum Fisher information on the ultimately chosen ranked set sample.

Let $Y_{r:[n]}, r=1,2, \ldots, n$ be the observations of the OUERSS arising from MTBED with pdf defined in (3.1) obtained by ordering the the observations $Y_{[n] i} i=1,2, \ldots, n$ as ascending order of magnitude. Then from (2.7) and (2.8) we obtain the moments and the product moments of $Y_{r:[n]}$ for $r=1,2, \ldots, n$ as given below.

For $1 \leq r \leq n, q \geq 1$

$$
\begin{aligned}
E\left[Y_{r:[n]}^{q}\right]=\theta_{2}^{q} & \frac{n !}{(r-1) !(n-r) !} \sum_{r=0}^{n-r} \sum_{l=0}^{r+k-1}(-1)^{k+l}\left(\begin{array}{c}
n-r \\
k
\end{array}\right)\left(\begin{array}{c}
r+k-1 \\
s
\end{array}\right) \\
& {\left[\alpha_{n}^{l} \beta(r+k, l+1) \mu_{r+k: r+k+l}^{(q)}+\alpha_{n}^{l+1} \beta(r+k+1, l+1) \mu_{r+k+1: r+k+l+1}^{(q)}\right.} \\
& \left.+\alpha_{n}^{l+1} \beta(r+k, l+1) \mu_{r+k: r+k+l+1}^{(q)}\right] \\
& =\theta_{2}^{q} \mu_{r:[n]}^{(q)}(\text { say })
\end{aligned}
$$

and for $1 \leq r<s \leq n$,

$$
\begin{aligned}
& E\left[Y_{r:[n]} Y_{s:[n]}\right]=\theta_{2}^{2} \frac{n !}{(r-1) !(s-r-1) !(n-r) !} \sum_{k=0}^{s-r-1} \sum_{m=0}^{k} \sum_{l=0}^{r-1} \sum_{t=0}^{n-s}(-1)^{l+k+m} \\
& \left(\begin{array}{c}
s-r-1 \\
k
\end{array}\right)\left(\begin{array}{c}
k \\
m
\end{array}\right)\left(\begin{array}{c}
r-1 \\
l
\end{array}\right)\left(\begin{array}{c}
n-s \\
t
\end{array}\right) \\
& {\left[\sum_{p=0}^{l+m} \sum_{q=0}^{k-m+t}(-1)^{p+q}\left(\begin{array}{c}
l+m \\
p
\end{array}\right)\left(\begin{array}{c}
k-m+t \\
q
\end{array}\right) \alpha_{n}^{l+k+t}\left(1-\alpha_{n}\right)\right.} \\
& \frac{(r+m+p-1) !(s-r-k-1) !(n-s-m+q+k) !}{(n+p+q) !} \\
& \times\left(\left(1-\alpha_{n}\right) \mu_{r+m+p, s+m+p-k: n+p+q}\right. \\
& \left.+2 \alpha_{n} \frac{r+m+p}{n+p+q+1} \mu_{r+m+p+1, s+m+p-k: n+p+q+1}\right) \\
& +\sum_{p=0}^{l+m} \sum_{q=0}^{k-m+t+1}(-1)^{p+q}\left(\begin{array}{c}
l+m \\
p
\end{array}\right)\left(\begin{array}{c}
k-m+t+1 \\
q
\end{array}\right) 2 \alpha_{n} \\
& \frac{(r+m+p-1) !(s-r-k-1) !(n-s-m+q+k) !}{(n+p+q) !} \\
& \times\left(\left(1-\alpha_{n}\right) \mu_{r+m+p, s+m+p-k: n+p+q}\right. \\
& \left.\left.+2 \alpha_{n} \frac{r+m+p}{n+p+q+1} \mu_{r+m+p+1, s+m+p-k: n+p+q+1}\right)\right] \text {, } \\
& =\theta_{2}^{2} \mu_{r, s:[n]}(\text { say }) \text {. }
\end{aligned}
$$


Since the marginal distribution of $Y$ is exponential distribution with pdf (3.2), $\mu_{r: n}^{(q)}$ is the qth moment of the $r t h$ order statistic and $\mu_{r, s: n}$ is the product moment of $r t h$ and sth order statistics of a random sample of size $n$ arising from the standard exponential distribution and are given below.

$$
\begin{gathered}
\mu_{r: n}^{(1)}=\sum_{k=r}^{n} \frac{1}{n-k+1} \\
\mu_{r: n}^{(2)}=\sum_{k=r}^{n} \frac{1}{(n-k+1)^{2}}+\left(\sum_{k=r}^{n} \frac{1}{n-k+1}\right)^{2}
\end{gathered}
$$

and

$$
\mu_{r, s: n}=\sum_{k=r}^{n} \frac{1}{(n-k+1)^{2}}+\left(\sum_{k=r}^{n} \frac{1}{n-k+1}\right)\left(\sum_{k=s}^{n} \frac{1}{n-k+1}\right) .
$$

Thus the means, variances and covariances of OUERSS are given by

$$
\operatorname{Var}\left[Y_{r:[n]}\right]=\left(\mu_{r:[n]}^{(2)}-\left(\mu_{r:[n]}^{(1)}\right)^{2}\right) \theta_{2}^{2}
$$

and

$$
\operatorname{Cov}\left[Y_{r:[n]}, Y_{s:[n]}\right]=\left(\mu_{r, s:[n]}-\mu_{r:[n]} \mu_{s:[n]}\right) \theta_{2}^{2}, r \neq s
$$

where $\mu_{r:[n]}=\mu_{r:[n]}^{(1)}$. Let us denote

$$
\begin{gathered}
\xi_{r}=\mu_{r:[n]}, \\
\delta_{r, r}=\mu_{r:[n]}^{(2)}-\left(\mu_{r:[n]}\right)^{2},
\end{gathered}
$$

and

$$
\delta_{r, s}=\mu_{r, s:[n]}-\mu_{r:[n]} \mu_{s:[n]} .
$$

Let $\mathbf{Y}_{[n]}=\left(Y_{1:[n]}, Y_{2:[n]}, \cdots, Y_{n:[n]}\right)^{\prime}$ and if the parameter $\alpha$ involved in $\xi_{r}$ and $\delta_{r, s}$ is known then proceeding as in [7] the BLUE $\hat{\theta}_{2}$ of $\theta_{2}$ is obtained as

$$
\hat{\theta}_{2}=\left(\xi^{\prime} G^{-1} \xi\right)^{-1} \xi^{\prime} G^{-1} \mathbf{Y}_{[n]}
$$

and

$$
\operatorname{Var}\left(\hat{\theta}_{2}\right)=\left(\xi^{\prime} G^{-1} \xi\right)^{-1} \theta_{2}^{2},
$$

where $\xi=\left(\xi_{1}, \xi_{2}, \cdots, \xi_{n}\right)^{\prime}$ and $G=\left(\left(\delta_{r, s}\right)\right)$. Clearly $\hat{\theta}_{2}$ as given in (3.6) can be written as $\hat{\theta}_{2}=\sum_{r=1}^{n} a_{r} Y_{r:[n]}$. 
Remark 3.1. As the association parameter $\alpha$ in (1.1) is involved in the BLUE $\hat{\theta}_{2}$ of $\theta_{2}$ and our assumption is $\alpha$ is known, one can obtain an estimate of $\alpha$ as given in [5].

We have computed the coefficients $a_{r}$ involved in $\hat{\theta}_{2}$ for $\alpha=0.25(0.25) 1.0 ; n=2(2) 10$ and are given in Table 1. [5] obtained the BLUE $\theta_{2}^{*}$ of the parameter $\theta_{2}$ using usual RSS and the BLUE $\tilde{\theta}_{2}$ of the parameter $\theta_{2}$ using ERSS. We have evaluated the efficiency $e\left(\hat{\theta}_{2} \mid \theta_{2}^{*}\right)=\frac{\operatorname{Var}\left(\theta_{2}^{*}\right)}{\operatorname{Var}\left(\hat{\theta}_{2}\right)}$ of $\hat{\theta}_{2}$ relative to $\theta_{2}^{*}$ and $e\left(\hat{\theta}_{2} \mid \tilde{\theta}_{2}\right)=\frac{\operatorname{Var}\left(\tilde{\theta}_{2}\right)}{\operatorname{Var}\left(\hat{\theta}_{2}\right)}$ of $\hat{\theta}_{2}$ relative to $\tilde{\theta}_{2}$ and are given in Table 2. From the Table 1, one can easily see that $\hat{\theta}_{2}$ is relatively more efficient than $\theta_{2}^{*}$ and $\tilde{\theta}_{2}$.

As mentioned earlier, for MTBED the concomitant of smallest order statistic possess the maximum Fisher information on $\theta_{2}$ whenever $\alpha<0$. Therefore when $\alpha<0$ we consider an OLERSS in which from each set we choose an unit of a sample with the smallest value on the auxiliary variable as the units of ranked sets with an objective of exploiting the maximum Fisher information on the ultimately chosen ranked set sample. Let $Y_{r:[1]}, r=1,2, \ldots, n$ be the observations of the OLERSS. Then due to the Remark 2.1 we have the pdf of $Y_{r:[1]}$ and the joint pdf of $Y_{r:[1]}$ and $Y_{s:[1]}$ for $\alpha<0$ is identically equal to the pdf of $Y_{r:[n]}$ and joint pdf of $Y_{r:[n]}$ and $Y_{s:[n]}$ for $\alpha>0$. Consequently we have $E\left(Y_{r:[n]}\right)$ for $\alpha>0$ and $E\left(Y_{r:[1]}\right)$ for $\alpha<0$ are identically equal. Similarly $\operatorname{Var}\left(Y_{r:[n]}\right)$ for $\alpha>0$ and $\operatorname{Var}\left(Y_{r:[1]}\right)$ for $\alpha<0$ are identically equal. Consequently if $\hat{\theta}_{2}^{(1)}$ is the BLUE of $\theta_{2}$, involved in MTBED for $\alpha<0$, based on the OLERSS observations $Y_{r:[1]}, r=1,2, \ldots, n$, then the coefficients of $Y_{r:[1]}, r=1,2, \ldots, n$ in the BLUE $\hat{\theta}_{2}^{(1)}$ for $\alpha<0$ is same as the coefficients of $Y_{r:[n]}, r=1,2, \ldots, n$ in the BLUE $\hat{\theta}_{2}$ for $\alpha>0$ based on OUERSS. Further we have $\operatorname{Var}\left(\hat{\theta}_{2}^{(1)}\right)=\operatorname{Var}\left(\hat{\theta}_{2}\right)$.

\section{Conclusion}

Ranked set sampling is applicable whenever ranking of a set of sampling units can be done easily by a judgment method or other inexpensive methods. Lots of work are available in literature on

Table 1. Coefficients of the estimators $\hat{\theta}_{2}$ of $\theta_{2}$ involved in Morgenstern type bivariate exponential distribution.

\begin{tabular}{|c|c|c|c|c|c|c|c|c|c|c|c|}
\hline$n$ & $\alpha$ & $a_{1}$ & $a_{2}$ & $a_{3}$ & $a_{4}$ & $a_{5}$ & $a_{6}$ & $a_{7}$ & $a_{8}$ & $a_{9}$ & $a_{10}$ \\
\hline \multirow[t]{4}{*}{2} & 0.25 & 0.4681 & 0.4841 & & & & & & & & \\
\hline & 0.5 & 0.4414 & 0.4685 & & & & & & & & \\
\hline & 0.75 & 0.4193 & 0.4534 & & & & & & & & \\
\hline & 1 & 0.4013 & 0.4385 & & & & & & & & \\
\hline \multirow[t]{4}{*}{4} & 0.25 & 0.2052 & 0.2247 & 0.2359 & 0.2366 & & & & & & \\
\hline & 0.5 & 0.1732 & 0.2085 & 0.2245 & 0.2224 & & & & & & \\
\hline & 0.75 & 0.1533 & 0.1992 & 0.2143 & 0.2076 & & & & & & \\
\hline & 1 & 0.1451 & 0.1945 & 0.2040 & 0.1927 & & & & & & \\
\hline \multirow[t]{4}{*}{6} & 0.25 & 0.1233 & 0.1376 & 0.1480 & 0.1546 & 0.1576 & 0.1556 & & & & \\
\hline & 0.5 & 0.0930 & 0.1212 & 0.1379 & 0.1464 & 0.1485 & 0.1436 & & & & \\
\hline & 0.75 & 0.0766 & 0.1156 & 0.1333 & 0.1395 & 0.1387 & 0.1312 & & & & \\
\hline & 1 & 0.0762 & 0.1186 & 0.1306 & 0.1319 & 0.1280 & 0.1189 & & & & \\
\hline \multirow[t]{4}{*}{8} & 0.25 & 0.0852 & 0.0959 & 0.1041 & 0.1104 & 0.1147 & 0.1173 & 0.1179 & 0.1157 & & \\
\hline & 0.5 & 0.0568 & 0.0795 & 0.0943 & 0.1034 & 0.1085 & 0.1105 & 0.1097 & 0.1054 & & \\
\hline & 0.75 & 0.0423 & 0.0759 & 0.0926 & 0.1003 & 0.1033 & 0.1032 & 0.1008 & 0.095076 & & \\
\hline & 1 & 0.0467 & 0.0838 & 0.0947 & 0.0975 & 0.0971 & 0.0950 & 0.0913 & 0.0850 & & \\
\hline \multirow[t]{4}{*}{10} & 0.25 & 0.0638 & 0.0720 & 0.0786 & 0.0839 & 0.0880 & 0.0910 & 0.0931 & 0.0941 & 0.0939 & 0.0919 \\
\hline & 0.5 & 0.0371 & 0.0559 & 0.0687 & 0.0773 & 0.0828 & 0.0861 & 0.0877 & 0.0879 & 0.0866 & 0.0831 \\
\hline & 0.75 & 0.0236 & 0.0531 & 0.0685 & 0.0764 & 0.0803 & 0.0820 & 0.0820 & 0.0809 & 0.0786 & 0.0743 \\
\hline & 1 & 0.0311 & 0.0642 & 0.0739 & 0.0768 & 0.0774 & 0.0768 & 0.0754 & 0.0733 & 0.0704 & 0.0658 \\
\hline
\end{tabular}


Table 2. Efficiencies of the estimators $\hat{\theta}_{2}$ relative to $\theta_{2}^{*}$ and $\tilde{\theta}_{2}$ of $\theta_{2}$ involved in Morgenstern type bivariate exponential distribution.

\begin{tabular}{rrrrrrr}
\hline$n$ & $\alpha$ & $\operatorname{Var}\left(\hat{\theta}_{2}\right)$ & $\operatorname{Var}\left(\theta_{2}^{*}\right)$ & $\operatorname{Var}\left(\tilde{\theta}_{2}\right)$ & $e_{1}$ & $e_{2}$ \\
\hline 2 & 0.25 & 0.4781 & 0.4793 & 0.4988 & 1.0025 & 1.0433 \\
& 0.5 & 0.4574 & 0.4586 & 0.4950 & 1.0026 & 1.0822 \\
& 0.75 & 0.4361 & 0.4383 & 0.4878 & 1.0050 & 1.1185 \\
& 1 & 0.4159 & 0.4184 & 0.4748 & 1.0060 & 1.1416 \\
\hline 4 & 0.25 & 0.2212 & 0.2313 & 0.2494 & 1.0456 & 1.1274 \\
& 0.5 & 0.2029 & 0.2131 & 0.2475 & 1.0503 & 1.2098 \\
& 0.75 & 0.1853 & 0.1957 & 0.2439 & 1.0561 & 1.3162 \\
& 1 & 0.1687 & 0.1790 & 0.2374 & 1.0610 & 1.3262 \\
\hline 6 & 0.25 & 0.1417 & 0.1519 & 0.1663 & 1.0720 & 1.1736 \\
& 0.5 & 0.1273 & 0.1376 & 0.1650 & 1.0809 & 1.2961 \\
& 0.75 & 0.1137 & 0.1240 & 0.1626 & 1.0906 & 1.4301 \\
& 1 & 0.1011 & 0.1113 & 0.1583 & 1.1009 & 1.5658 \\
\hline 8 & 0.25 & 0.1028 & 0.1130 & 0.1247 & 1.0992 & 1.2130 \\
& 0.5 & 0.0911 & 0.1014 & 0.1238 & 1.1131 & 1.3589 \\
& 0.75 & 0.0801 & 0.0904 & 0.1220 & 1.1285 & 1.5231 \\
& 1 & 0.0710 & 0.0802 & 0.1187 & 1.1295 & 1.6718 \\
\hline 10 & 0.25 & 0.0797 & 0.0899 & 0.0998 & 1.1279 & 1.2521 \\
& 0.5 & 0.0699 & 0.0802 & 0.0990 & 1.1473 & 1.4163 \\
& 0.75 & 0.0618 & 0.0710 & 0.0976 & 1.1489 & 1.5793 \\
& 1 & 0.0542 & 0.0625 & 0.0940 & 1.1531 & 1.7343 \\
\hline & & & & & &
\end{tabular}

theory and applications of ranked set sampling when ranking is perfect. In this work we considered ranked set sampling when ranking is imperfect, in which an auxiliary variable $X$, correlated with the study variate $Y$, is used to rank the units in each set, under the assumption that $(X, Y)$ follows MFD. We considered a modified ranked set sampling procedure called ordered extreme ranked set sampling, which is attained by ordering the units in the extreme ranked set sample. The distribution theory thus obtained was used to estimate a parameter associated with the study variate $Y$ when $(X, Y)$ follows a MTBED. We obtained the coefficients of the BLUE and its variance numerically for different choices for $n$, the sample size and $\alpha$, the association parameter between $X$ and $Y$. We obtained an efficiency comparison of the proposed estimator with estimators based on usual RSS and ERSS and found that estimator based on OERSS is more efficient that usual RSS and ERSS. Moreover the efficiency based on OERSS increases with $n$ and $\alpha$ which is expected.

\section{References}

[1] Z.A. Abo-Eleneen and H.N. Nagaraja, Fisher information in an order statistic and its concomitant, Annals of Institute of Statistical Mathematics 54 (2002) 667-680.

[2] L.J. Bain, Statistical analysis of reliability and life testing models: theory and methods (Marcel Dekker, New York, 1978).

[3] V. Barnett and K. Moore, Best linear unbiased estimates in ranked-set sampling with particular reference to imperfect ordering, Journal of Applied Statistics 24 (1997) 697-710.

[4] M. Chacko, and P. Y. Thomas, Estimation of a parameter of bivariate Pareto distribution by ranked set sampling, Journal of Applied Statistics 34 (2007) 703-714.

[5] M. Chacko, and P. Y. Thomas, Estimation of parameters of Morgenstern type bivariate exponential distribution by ranked set sampling, Annals of the Institute of Statistical Mathematics 60 (2008) 301318. 
[6] M. Chacko, and P. Y. Thomas, Estimation of parameters of Morgenstern type bivariate logistic distribution by ranked set sampling, Journal of the Indian Society for Agricultural Statistics 63 (2009) $77-83$

[7] H.A. David and H.N. Nagaraja, Order statistics., (John Wiley and Sons, New York, 2003).

[8] S. Kotz, N. Balakrishnan and N.L. Johnson, Distributions in statistics: continuous multivariate distributions, (John Wiley and Sons, New York, 2000).

[9] K. Lam, B.K. Sinha and Z. Wu, Estimation of a two-parameter exponential distribution using ranked set sample, Annals of the Institute of Statistical Mathematics 46 (1994) 723-736.

[10] K. Lam, B.K. Sinha and Z. Wu, Estimation of location and scale parameters of a logistic distribution using ranked set sample. In H.N. Nagaraja, P. K. Sen and D.F. Morrison(Ed.) Statistical theory and applications: papers in honor of Herbert A. David, (Springer, New-York, 1996).

[11] G. A. McIntyre, A method for unbiased selective sampling, using ranked sets, Australian Journal of Agricultural Research 3 (1952) 385-390.

[12] J. Scaria and N.U. Nair, On concomitants of order statistics from Morgenstern family, Biometrical Journal 41 (1999) 483-489.

[13] S. L. Stokes, Ranked set sampling with concomitant variables, Communications in Statistics; Theory and Methods 6 (1977) 1207-1211.

[14] S. L. Stokes, Inference on the correlation coefficient in bivariate normal populations from ranked set samples, Journal of the American Statistical Association 75 (1980) 989-995.

[15] S. L. Stokes, Parametric ranked set sampling, Annals of the Institute of Statistical Mathematics 47 (1995) 465-482. 